Pre-print from: Saulynas, S. \& Kuber, R. 2018: Towards Supporting Mobile Device Users Facing Severely Constraining Situational Impairments. In Extended Abstracts of the 2018 CHI Conference on Human Factors in Computing Systems - CHI '18, Montreal, Canada, LBW540.

\title{
Towards Supporting Mobile Device Users Facing Severely Constraining Situational Impairments
}

\author{
Sidas Saulynas \& Ravi Kuber \\ UMBC \\ Baltimore, MD 21250, USA \\ \{ saulyn1, rkuber\}@umbc.edu
}

\begin{abstract}
A subset of "Situationally Induced Impairments and Disabilities" (SIID), termed "Severely Constraining Situational Impairments" (SCSI), was explored at the user task and motivational level, to better understand the challenges faced by users attempting to perform tasks using a mobile device. Through structured interviews, participants were found to deploy workarounds in attempting to complete mobile 1/O transactions, even if that workaround might place them in considerable danger. The motivations underlying user decisions were also explored resulting in a set of rich scenarios which will be used in the final participatory design stage of the study to discover ways that technology can be designed to overcome SCSIs.
\end{abstract}

\section{Author Keywords}

SIID; Situational Impairments; SCSI; Mobile devices.

\section{Introduction}

Interactions with mobile devices can be negatively impacted by situational, contextual or environmental factors (termed "Situationally Induced Impairments and Disabilities" (SIID) [1]). Examples include sunlight causing glare on a screen, affecting the ability to read an SMS. Under more challenging circumstances (i.e., when one or more channels are blocked, restricted or overloaded, or when the hands are encumbered), further difficulties may be faced attempting to perform tasks using a mobile device (termed "Severely Constraining Situational Impairments" (SCSI)). These are often so overwhelming that the multitude and complexity of the ambient agents lead to the inability to devise a solution or reasonable workaround [2]. An example of a SCSI is shown in Figure 1.

A user wants to look something up on the Web while mowing their lawn. They cannot immediately access their device because (1) their hands are not free, (2) the ambient noise is preventing voice input, and (3) the bright sunlight is preventing accurate readout of the output even if (1) and (2) can be addressed. The issue is further exacerbated due to cognitive load. By the time the user has effectively achieved a context where (1), (2), and (3) are mitigated, the user may not need to perform the task or may have forgotten the purpose of the original task.

Figure 1: An example of a "Severely Constraining Situational Impairment" (SCSI)

While SCSIs were first described in detail by [2], to our knowledge, there has been no additional research to date examining how this complex and rapidly changing situational impairment phenomenon 
Pre-print from: Saulynas, S. \& Kuber, R. 2018: Towards Supporting Mobile Device Users Facing Severely Constraining Situational Impairments. In Extended Abstracts of the 2018 CHI Conference on Human Factors in Computing Systems - CHI '18, Montreal, Canada, LBW540.

is being attended to by users. For example, as the result of limitations of existing technology to account for interaction needs in the variable contexts where SCSIs reside, are users abandoning the transaction attempts or employing workarounds? Also, what are user motivations for either voluntarily foregoing a transaction attempt or attempting the transaction using a workaround even when such action may be frowned upon or dangerous?

If users are attempting to overcome transaction issues by developing workarounds, an unsuccessful transaction attempt as the result of the presence of a SCSI might be an inconvenience or loss of productivity. However, the desire to complete a transaction in spite of the contextual limitations could also place the user at both physical risk as well as cultural risk if their actions are in violation of a societal law or norm (e.g., checking a mobile device while driving).

This study intends to add a piece to this puzzle in a region of the problem space that has yet to be properly charted. A set of interviews were conducted to better understand the nature of SCSIs, and how these differ from SIIDs when performing mobile interactions. Strategies/workarounds for challenging circumstances were also investigated. By being able to better understand the area, we can then identify ways in which technology may be able to help support the user when performing mobile tasks, even if constraints from the situation or environment are present.

\section{Related Work}

To discover SIID themes, Saulynas et al. [2] conducted a diary study which resulted in a corpus of "Situational Impairment Events" (SIEs). Phenomenological analysis on the SIE corpus resulted in the creation of five SIID classification themes. The creation of categories highlighting the similarities and/or differences that users of mobile technology experience might prove helpful in the formulation of future mobile design guidelines. In a later study, Sarsenbayeva et al. [3] provided a systematic overview of "established situational impairments", their impact on mobile device interaction, and suggested methods for their detection and design guidelines.

Other studies such as those conducted by Goel et al. [4] have focused on particular encumbrances associated with SIIDs and explored possible design solutions. Lin et al. [5] attempted to measure and quantify the effects of variable context on input performance. These studies examined SIIDs as singular impairments or focused on a specific aspect for design to address. However, given the rapid adoption and embedding of smartphones into everyday life, exploring SIIDs as single events in the rich, variable, and complex context in which they exist, can be limiting.

In [2], SCSIs were found to be distinguishable from SIIDs based on several characteristics. For example, a solution or workaround may not be found in sufficient time for the transaction to have any value to the user. Also, multiple impairment events might be attempting to occupy the same transaction space, or a solution to an initial SIID may result in the creation of a new SIID. The study identified the existence of SCSIs, but in many cases the reasons as to why a transaction was postponed or abandoned was not 
Pre-print from: Saulynas, S. \& Kuber, R. 2018: Towards Supporting Mobile Device Users Facing Severely Constraining Situational Impairments. In Extended Abstracts of the 2018 CHI Conference on Human Factors in Computing Systems - CHI '18, Montreal, Canada, LBW540.

revealed in the data. Understanding the motivation behind a user action can be an important component in designing for an effective user experience. Furthermore, when a workaround was deployed, understanding the reasons why a particular series of steps were used would offer insights into the interaction challenges faced by users. Finally, if multiple SIEs were present at once, we aimed to examine the demands faced by users to account for these. The present study builds on the findings implied by the discovery of the SCSI subset by examining how user motivations and workaround attempts influenced the overall mobile transaction experience during a SCSI event.

\section{Methodology and Research Questions}

Three research questions are addressed in two stages.

\section{Research Questions}

- RQ1: Do users deploy workarounds when attempting to complete a mobile I/O transaction in the presence of a SCSI?

- RQ2: What are the motivations behind users choosing to forego the completion of a mobile I/O transaction or choosing to attempt transaction completion even in contexts that represent a violation of a cultural rule or standard or even physical danger?

- RQ3: What are the workaround tasks which users deploy in the presence of a SCSI, and can user workaround tasks and motivations be accounted for when designing for mobile transactions?

\section{Stage 1: Interview}

In order to address RQ1 and RQ2, and to confirm the findings in [2], Stage 1 will consist of interviews with mobile device users. Structured questions explore what are common information activities which users may perform using a mobile device, particularly when situational, contextual or environmental factors are present impacting the interaction. We aim to shed light upon the internal decision making processes leading to execution of task steps. Data from Stage 1, once coded and analyzed, will help confirm or modify which tasks appear to be the most common that might be affected by the onset of a SCSI as well as the tasks that can be differentiated as SCSI vs. SIID. The culmination of this stage will be the construction of three to five representative SIID and SCSI scenarios.

\section{Stage 2: Participatory Design}

To address RQ3, Stage 2 will engage mobile device users and interface designers using a participatory design approach to discover ways that technology can be designed to overcome the SCSIs represented in the Stage 1 scenarios. These scenarios will hopefully reveal the role of mobile transaction modalities in contributing to a better user experience as well as allow the team to brainstorm design ideas. The researcher will lead the discussion of the main issues covered by the scenarios, allowing design suggestions and idea reflection. 
Pre-print from: Saulynas, S. \& Kuber, R. 2018: Towards Supporting Mobile Device Users Facing Severely Constraining Situational Impairments. In Extended Abstracts of the 2018 CHI Conference on Human Factors in Computing Systems - CHI '18, Montreal, Canada, LBW540.

\section{Participants and Coding Strategy for Stage 1}

Twenty participants (seven females), with ages ranging from 19 to 66 (mean: 28.6) were recruited by direct invitation. Each was presented with a series of interview questions. After completion of each interview, data was transcribed and coded by two researchers. Inter-rater reliability tests were then performed to indicate level of agreement among codes.

\section{Preliminary Findings from Stage 1}

\section{Interview Analysis}

In the first interview segment, participants were asked to list tasks that they performed using their smartphones on a daily basis, then approximate how many times per day they engage in each task performed on their device (i.e., sending/receiving SMS messages - 50 times/day). The data was tallied resulting in an estimated collection of 3,452 daily events (mean: 172.6). By far the most frequent task was SMS/messaging ( $40.8 \%$ of the estimated daily smartphone activity). Combined with other tasks in the top five (social media, email, Internet browsing, and streaming media), these categories represented $78.9 \%$ of the total. The top heavy concentration of task types proved a significant factor in creating the scenarios.

The SIID category in [2] identified as "Social/Cultural Issues" (no physical barrier to transaction completion but nevertheless can hinder effective transaction completion) was sub-classified into one of three subcategories (detailed in Figure 2). An important segment of the interview was to confirm these sub-categories as representing underlying motivations to voluntarily forego transaction completion. In addition, since motivation could be an important factor in classifying an event as a SCSI or SIID (i.e. the time sensitivity of the information), confirming the why in addition to the what is needed. Participants were asked, if they ever wanted to interact with their smartphone using their hands while in legally- or socially-unacceptable contexts (i.e., driving, on public transport, or at a public performance) but consciously chose not to. All 20 participants indicated, for most or all of the offered contexts, that they did. Then they were asked for the reasons they chose to forego or abandon the transactions, and their responses were recorded and coded. Some responses reflected concern for social inappropriateness. For example, in response to why they chose to forego smartphone usage on public transport, one participant noted that they did not want to "feel like a zombie...like everyone else." Others noted safety concerns. For example, one user noted: "accident, death, not seeing my wife, not seeing my children" as the reasons they chose to not interact with their smartphone while driving.

1. Fear of Reprisal from an Authority (i.e., a citation from a police officer or reprimand from a boss/teacher)

2. Safety (i.e., concern over the potential harm to oneself or others)

3. Socially Acceptable Behavior (i.e., where neither reprisal nor safety were the primary motivation, but rather a sense by the user that engaging in the transaction was simply not the correct thing to do in a given social context)

Figure 2: Social/Cultural Issue Subcategories (as defined in [2]). 
Pre-print from: Saulynas, S. \& Kuber, R. 2018: Towards Supporting Mobile Device Users Facing Severely Constraining Situational Impairments. In Extended Abstracts of the 2018 CHI Conference on Human Factors in Computing Systems - CHI '18, Montreal, Canada, LBW540.

No response was determined to be outside of the three sub-categories defined in [2] and confirmed when analyzed for inter-rater reliability. Each context was analyzed separately with all calculating to a Cohen's Kappa score above 0.6 indicating good agreement among reviewers. Because the responses helped reveal user motivation, these findings help substantiate RQ2.

Perhaps the most interesting and potentially significant finding, however, was after each context question, the participants were asked, despite the reasons they indicated as to why they chose to forego a transaction, did they ever "override" those rules and attempt a transaction anyway. For every context that was applicable to the participant, $100 \%$ indicated that they have overridden their own rules. The same participant, for example, that offered the histrionic "accident, death..." response in the above example, when asked if they ever did it anyway said, without a moment's hesitation, "oh yeah!" These findings not only substantiate both RQ1 and RQ2, but also highlight the importance that smartphone users currently place on transaction completion.

Finally, participants were asked whether they ever engaged in transaction forbearance due to the issues defined as the four characteristics of a SCSI and/or if they attempted workarounds to the limitations they faced. For example, in response to the question of whether they ever wanted to interact with their smartphone, could not, and by the time circumstances allowed interaction, the reason for the interaction became meaningless, one participant noted: "Text message(s) not going through...to...my boyfriend in the store to get me something. By the time the message goes through he's back in the car." In response to a question of multiple impairment events occupying the same transaction space one participant noted: "Using my GPS and got a text and missed the exit". These responses were recorded, coded for each SCSI sub-category, and analyzed for inter-rater reliability. A Cohen's Kappa score 0.67 confirmed good agreement among reviewers. The results also substantiate RQ1.

\section{SIID/SCSI Scenarios}

A set of five scenarios, each with at least one sub-scenario representing an SIID event and one representing a SCSI event were created. They were based on an amalgamation of the common tasks that were identified as well as being situated within the generalized conditions for potential SIE events that were found in [2]. Each set will be presented to participants in participatory workshops.

The purpose in creating the scenarios was (1) to produce common, recognizable situations that a group of smartphone users could easily identify with and use as a basis for stimulating ideas for design solutions and (2) help differentiate an SIID and a SCSI event more clearly. These preliminary results will now serve as a springboard for the final stage of this study. Two of the scenarios are detailed below. A brief overview of the other three appear in Figure 3. 
Pre-print from: Saulynas, S. \& Kuber, R. 2018: Towards Supporting Mobile Device Users Facing Severely Constraining Situational Impairments. In Extended Abstracts of the 2018 CHI Conference on Human Factors in Computing Systems - CHI '18, Montreal, Canada, LBW540.

\section{SCENARIO 1: WALKING (YOU ARE WALKING DOWN THE HALL AND YOUR PHONE VIBRATES IN YOUR POCKET)}

- SIID: You are not in a hurry to get anywhere nor are you expecting any important message or phone call.

- SCSI: You are already late for a meeting that you will be in trouble for if you are any later than you already are but this may be an important message that you have been waiting for so you are compelled to unlock the phone and read it. After reading it, not only do you confirm that this is the message that you were waiting for, but a timely response is required that you must compose and send without undue loss of time as you progress to your meeting. The message that you have to send is very long.

\section{SCENARIO 2: DRIVING}

- SIID: You are lost and need to access your maps application to get directions. You do not have a dashboard holder for your phone.

- SCSI: In addition to above, you cannot use voice interface because you need to authenticate entry to your phone and someone else is in the car with you that you do not trust. There is nowhere to pull over. You are running late and if you do not get directions ASAP you may miss an important meeting.

For the final (participatory design) stage of this study there may be multiple workshops held with a purpose of enabling each group to either strengthen ideas from the previous groups, or to provide new design suggestions which can be prototyped during each session.

- $\quad$ Scenario 3: Driving Continued -Navigation is interrupted by another task requesting modal attention (i.e., phone call)

- Scenario 4: Movie Theater -Watching a movie in a crowded theater and phone vibrates in pocket

- Scenario 5: At Home Performing Task - Making dinner...hands are full and messy... smartphone rings and is on the counter

Figure 3: Synopsis of Additional Scenarios

\section{Conclusion and Future Work}

This paper represents the first step in this research. The analysis and understanding of SCSIs is important from not only a UX perspective, but also a user and stakeholder safety perspective in the brave new and rapidly changing world of omnipresent information. This new interaction paradigm has truly become an important and embedded part of our lives to the point that the thou shalt not rules are understood but are in equal measure ignored. If we do not account for this need, then mobile design is putting users in danger, and therefore failing our users.

The interview stage identified common user smartphone tasks and begun an exploration of the internal motivational process that users attend to when attempting to overcome both SIIDs and SCSIs. The importance users place in completing some mobile tasks, regardless of the risk, has also been 
Pre-print from: Saulynas, S. \& Kuber, R. 2018: Towards Supporting Mobile Device Users Facing Severely Constraining Situational Impairments. In Extended Abstracts of the 2018 CHI Conference on Human Factors in Computing Systems - CHI '18, Montreal, Canada, LBW540.

uncovered. What still needs to be done is research an analysis that will help answer RQ3, specifically what are the workarounds that users are performing and how can these along with the emphatic knowledge gleaned through the understanding of motivation help influence mobile technology design. The rich user SIID and SCSI scenarios created will feed the final participatory design stage of this study in an attempt to answer RQ3.

\section{References}

1. Andrew Sears, Min Lin, Julie Jacko and Yan Xiao. 2003. When computers fade: pervasive computing and situationally-induced impairments and disabilities. In Proceedings of $\mathrm{HCl}$ International, 12981302.

2. Sidas Saulynas, Lawrence. E. Burgee and Ravi Kuber. 2017. All situational impairments are not created equal: a classification system for situational impairment events and the unique nature of severely constraining situational impairments. In Proceedings of iConference.

3. Zhanna Sarsenbayeva, Niels van Berkel, Chu Luo, Vassilis Kostakos and Jorge Goncalves. 2017. Challenges of Situational Impairments during Interaction with Mobile Devices. In Proceedings of the 29th Australian Conference on Computer-Human Interaction (OZCHI'17), 477-481. http://doi.acm.org/10.1145/3152771.3156161

4. Mayank Goel, Leah Findlater and Jacob Wobbrock. 2012. WalkType: using accelerometer data to accommodate situational impairments in mobile touch screen text entry. In Proceedings of the SIGCHI Conference on Human Factors in Computing Systems (CHI'12), 2687-2696. http://doi.acm.org/10.1145/2207676.2208662

5. Min Lin, Rich Goldman, Kathleen J. Price, Andrew Sears and Julie Jacko. 2007. How do people tap when walking? An empirical investigation of nomadic data entry. International Journal of HumanComputer Studies, 65, 9, 759-769. https://doi.org/10.1016/j.ijhcs.2007.04.001 